\title{
SEMI-TRANSLATION PLANES(1)
}

\author{
BY \\ T. G. OSTROM
}

I. Introduction. The known finite projective planes are all either in one of the following three classes or are dual to planes in one of these classes. The three classes are: (1) the translation planes, (2) the Hughes planes, (3) a class of planes constructed by the author. Classes (2) and (3) all come under the heading of what we call "semi-translation planes" in the definition given below. $\S \S$ II and IV of this paper constitute a general investigation of the properties of semi-translation planes, their coordinate systems and collineations.

In [8], the author developed a method of constructing translation planes by using appropriately selected sets of points in a Desarguesian plane as the lines of a translation plane. In [9], this method was adapted to obtain a new class of planes from dual translation planes. Hughes pointed out to the author and it was also noted by Albert that the same method can be applied to the Hughes planes. Thus all the known semi-translation planes not in class (1) are related to other planes by this construction process. Moreover, we show (in $\S \mathrm{V}$ ) that the planes obtained from the Hughes planes constitute a fourth class in addition to those mentioned in the first paragraph.

In §III, we make a further study of this construction process, putting it into a more general form. The question remains open as to whether this generalization will make it possible to obtain still more new planes and just how extensive the class of semi-translation planes really is.

The planes with which we are concerned are all of order $q^{2}$, where $q$ is a prime power. This condition is to be understood throughout unless we indicate to the contrary.

Definitions for the basic concepts used in projective and affine planes may be found in [6] or [11].

\section{Collineations and dualities.}

Definition 1. A projective plane $\pi$ of order $q^{2}(q>2)$ will be said to be a semi-translation plane with respect to the line $l$ if there exists a set $\mathfrak{M}$ of $q+1$ points on $l$ such that if $P \in \mathfrak{M}$, then $\pi$ admits a group of elations of order $q$ with center $P$ and axis $l$.

Presented to the Society, August 29, 1962; received by the editors August 13, 1962 and, in revised form, January 28, 1963.

(1) This work was supported (in part) by grant No. NSF-G16299 from the National Science Foundation. 
Definition 2. In Definition 1, if the total group of elations with axis $l$ is exactly of order $q^{2}$ (i.e., the elations mentioned constitute all of the elations with axis $l$ ) then $\pi$ will be said to be a strict semi-translation plane with respect to $l$.

Definition 3. The point $Q$ will be said to be a center for the line $m$ and $m$ will be said to be an axis for $Q$ if $Q \in M$ and $\pi$ admits a group of elations of order $q$ with center $Q$, axis $m$.

THEOREM 1. Let $\pi$ be a projective plane of order $n$ ( $n$ not necessarily a square). If for some line $l, \pi$ admits a group $G$ of elations with axis $l$, where the order of $G$ is greater than $n$, then every point $P$ on $l$ is the center of a nontrivial elation with axis $l$.

Proof. Let $\pi^{*}$ be the affine plane obtained by deleting $l$ from $\pi$. Then $G$ partitions the points of $\pi^{*}$ into transitive classes of length $k$, where $k$ is the order of $G$. If $P \in l$, and $k>n$, then at least one of the $n$ affine lines through $P$ must contain at least two points $R$ and $S$ in the same transitive class. The translation (elation with axis $l$ ) which carries $R$ into $S$ must have $P$ as its center.

LEMMA 1. Let $\pi$ be a semi-translation plane with respect to $l$. Let $G$ be a group of elations with axis $l$ such that $G$ is of order $q^{2}$ and for each point $P \in \mathfrak{M}$, the subgroup of $G$ with center $P$ is of order $q$. Let $\mathfrak{I}$ be a transitive class under $G$ of points not on $l$. Then $T \cup \mathfrak{M}$ is the set of points in a projective subplane of order $q$.

Proof. Let the points of $\pi_{0}$ be the points in $\mathfrak{I} \cup \mathfrak{M}$. If $m$ is a line of $\pi$ which contains at least two points of $\pi_{0}$, let $m \cap \pi_{0}$ be a line of $\pi_{0}$. We must show that $\pi_{0}$ is a projective plane.

Since $\mathfrak{I}$ contains $q^{2}$ points and $\mathfrak{M}$ contains $q+1$ points, $\pi_{0}$ contains $q^{2}+q+1$ points. Two points of $\pi_{0}$ lie on exactly one line of $\pi_{0}$; each line of $\pi_{0}$ contains exactly $q+1$ points of $\pi_{0}$. It follows that each point lies on $q+1$ lines and finally that every two lines have a point in common. We are assuming $q>2$; there is at least one proper quadrangle in $\pi_{0}$. It follows that $\pi_{0}$ is a projective subplane.

LEMMA 2. If $\pi$ is a strict semi-translation plane with respect to $l_{1}$ and also with respect to $l_{2}$ and if $P=l_{1} \cap l_{2}$, then $P$ must be a center (Definition 3) for $l_{1}$ and $l_{2}$.

Proof. We shall make repeated use of the fact that if $\pi$ is a strict semi-translation plane with respect to $l$, if $P$ is a center for $l$ and if $\sigma$ is any collineation of $\pi$, then $\pi$ is a strict semi-translation plane with respect to $l \sigma$ and $P \sigma$ is a center for $l \sigma$.

Let $G\left(l_{1}\right)$ be the group of elations with axis $l_{1}$. By hypothesis, $G\left(l_{1}\right)$ is of order $q^{2}$. Let $Q$ be a point on $l_{1}$ which is not a center for $l_{1}$. Then the $q^{2}$ lines through $Q$ which are distinct from $l_{1}$ all lie in a single transitive class under $G\left(l_{1}\right)$.

The argument is by contradiction. If $P=l_{1} \cap l_{2}$ and $P$ is not a center for $l_{1}$, 
then $G\left(l_{1}\right)$ and $G\left(l_{2}\right)$ generate a group of collineations of $\pi$ which fixes $P$ and is transitive on all lines through $P$. Thus $\pi$ is a semi-translation plane (abbreviated s.t.) with respect to every line through $P$ and $P$ is not a center for any line through $P$.

Let $\mathbb{C}$ be the set of points $A$ such that $A$ is a center for $A P$. Since each of the $q^{2}+1$ lines through $P$ has exactly $q+1$ centers and $P$ is not a center, $\mathbb{C}$ contains exactly $\left(q^{2}+1\right)(q+1)$ points. Moreover $\mathbb{C}$ must be carried into itself by all collineations fixing $P$.

Let $E \neq P$ be a point not in $C$. Under the elation group with axis $E P$, the $q^{2}$ lines through $E$ which are distinct from $E P$ are all in the same transitive class. Hence these $q^{2}$ lines must each contain the same number, say $t$, of points in $\mathbb{C}$. There are $q+1$ points of $\mathbb{C}$ on EP; hence the total number of points in $\mathbb{C}$ is $q^{2} t+q+1=\left(q^{2}+1\right)(q+1)$. Hence $t=q+1$. Thus we have: If $E$ is not in $\mathbb{C}$, every line through $E$ contains exactly $q+1$ points of $\mathfrak{C}$. This in turn implies that if $l^{\prime}$ is any line of $\pi$, either every point of $l^{\prime}$ is in $\mathbb{C}$ or exactly $q+1$ points of $l^{\prime}$ are in $\mathbb{C}$.

Now let $A$ belong to $\mathfrak{C}$. If $k$ lines through $A$ contain $q^{2}+1$ points of $\mathfrak{C}$ then $q^{2}+1-k$ lines through $A$ contain $q+1$ points of $\mathfrak{C}$. It follows that $q^{2} k+q\left(q^{2}+1-k\right)+1=\left(q^{2}+1\right)(q+1)$, which reduces to $(q-1) k=q$.

For $q>2$, this equation has no solution in integers. The assumption that $P$ is not a center for $l_{1}$ thus leads to a contradiction. Since the roles of $l_{1}$ and $l_{2}$ can be interchanged, $P$ must be a center for both $l_{1}$ and $l_{2}$.

REMARK. We shall throughout this paper be interested in subplanes of order $q$. Henceforth, when we mention a subplane, it is to be understood that we mean a subplane of order $q$ unless indications are given to the contrary. We shall also find it convenient to use the expression " $l$ is a line of $\pi_{0}$ " in the case where $l$ is a line of $\pi$ such that $l \cap \pi_{0}$ is, strictly speaking, a line of $\pi_{0}$.

Lemma 3. Under the hypothesis of Lemma 2 there is a subplane $\pi_{0}$ such that:

(1) $\pi$ is a strict s.t. plane with respect to every line of $\pi$ which belongs to $\pi_{0}$ and goes through $P$.

(2) If $Q \in \pi_{0}, Q$ is a center for $Q P$.

Proof. Let $R$ be a point which is a center for $l_{2}$. By Lemma 1, the centers of $l_{1}$ together with the transitive class containing $R$ under the elation group $G\left(l_{1}\right)$ constitute the points of a subplane $\pi_{0}$.

By Lemma $2, P \in \pi_{0}$.

Now $P$ is fixed by $G\left(l_{1}\right)$ and the lines of $\pi_{0}$ which go through $P$ and are distrinct from $l_{1}$ lie in a single transitive class under $G\left(l_{1}\right)$. Part (1) follows. Since $R$ is a center for $R P=l_{2}$, it follows that if $Q \in \pi_{0}$, then $Q$ is a center for $Q P$ either by virtue of being a center for $l_{1}$ or by being an image of $R$ under some collineation in $G\left(l_{1}\right)$. Note that we have not established the uniqueness of $\pi_{0}$.

LEMMA 4. Under the hypothesis of Lemma $2, \pi$ is s.t. with respect to exactly $q+1$ lines through $P$. 
Proof. Let $\mathbb{C}$ be the set of points including $P$ and all points $A \neq P$ such that (1) $\pi$ is s.t. with respect to $A P,(2) A$ is a center for $A P$.

Suppose that $\pi$ is a (strict) s.t. plane with respect to exactly $k$ lines through $P$. Then $\mathbb{C}$ contains $k q+1$ points. Let $\mathbb{E}$ be the set of points $B$ such that $\pi$ is s.t. with respect to $B P$, but $B \notin \mathbb{C}$. Then the $q^{2}$ lines through $B$ distinct from $B P$ are all in the same transitive class under the group of elations with axis $B P$. Hence there is some integer $j$ such that each of these lines contains $j$ points in $\mathfrak{C}$. Counting the points on $B P$, the total number of points of $\mathbb{C}$ is therefore

and hence

$$
q^{2} j+q+1=k q+1
$$

$$
q j+1=k .
$$

Now any line not through $P$ which contains a point of $\mathbb{E}$ must contain exactly $j$ points of $\mathfrak{C}$. If $\pi$ is s.t. with respect to $l_{i}, i=1, \cdots, k$, then each point on $l_{i}$ belongs to either $\mathfrak{E}$ or $\mathfrak{C}$. Hence any line not through $P$ which contains no points of $\mathfrak{E}$ must contain $k$ points of $\mathfrak{C}$. Thus every line not through $P$ contains either $k$ or $j$ points of $\mathfrak{C}$. Now let $A \neq P, A \in \mathbb{C}$. If each line through $A$ different from $A P$ contains $j$ points of $\mathbb{C}(j-1$ points different from $A)$, then we must we have that the total number of points in $\mathbb{C}$ is

$$
(j-1) q^{2}+q+1=q^{2} j+q+1 .
$$

Since $q \neq 0$, this leads to a contradiction.

We have remaining the possibility that at least one line through $A$ contains $k$ points of $\mathfrak{C}$. Now the lines through $A$ (distinct from $A P$ ) occur in transitive classes of length $q$ under the elation group with axis $A P$. Hence if one line contains $k$ points, at least $q$ lines contain $k$ points of $C$. These $q$ lines together with $A P$ then account for all

$$
k q+1=(k-1) q+q+1 \text { points of } \mathfrak{C} .
$$

But the remaining $q^{2}-q$ lines through $A$ must each contain $j$ points of $\mathfrak{C}$. We have a contradiction unless $j=1$, and $k=q+1$.

LEMMA 5. If $\pi$ is a strict s.t. plane with respect to three nonconcurrent lines $l_{1}, l_{2}$, and $l_{3}$ and if $l_{1} \cap l_{2}=P, l_{2} \cap l_{3}=Q, l_{3} \cap l_{1}=R$, then there exists $a$ subplane $\pi_{0}$ which contains $P, Q$, and $R$ such that:

(1) $\pi$ is a strict s.t. plane with respect to every line of $\pi_{0}$ which goes through $P, Q$, or $R$.

(2) $P(Q$ or $R)$ is a center for every line of $\pi_{0}$ which goes through $P(Q$ or $R)$.

(3) If $T \in \pi_{0}, T \neq P, Q, R$ then $T$ is a center of $T P, T Q$, and $T R$.

Proof. Let $\pi_{1}, \pi_{2}, \pi_{3}$ be the subplanes determined by $l_{1}$ and $l_{2}, l_{2}$ and $l_{3}, l_{3}$ and $l_{1}$ as in Lemma 3. The points $P, Q$, and $R$ are centers for $l_{1}$ and $l_{2}, l_{2}$ and $l_{3}$ and $l_{1}$ by Lemma 2 . The subplanes $\pi_{1}$ and $\pi_{3}$ both contain the centers for $l_{1}$ and 
the transitive class which contains $Q$ under the elation group $G\left(l_{1}\right)$. Hence $\pi_{1}=\pi_{3}$. Similarly, $\pi_{1}=\pi_{2}$. Lemma 5 is then a direct consequence of Lemma 3 .

THEOREM 2. If $\pi$ is a strict semi-translation plane with respect to $l$ and $\pi$ admits a collineation moving $l$, then $\pi$ has a subplane $\pi_{0}$ of order $q$ such that:

(1) Every collineation of $\pi$ carries $\pi_{0}$ into itself.

(2) If $\pi$ is a strict semi-translation plane with respect to any line $l^{\prime}$, then $l^{\prime}$ belongs to $\pi_{0}$ and the centers of $l^{\prime}$ are points of $\pi_{0}$.

(3) Every point of $\pi_{0}$ is a center for some line of $\pi_{0}$.

Proof. Let $\sigma$ be a collineation displacing $l$. Let $\pi_{0}$ be the subplane determined by $l$ and $l \sigma$ as in Lemma 3. Let $P=l \cap l \sigma$. If $\pi$ admits a collineation $\rho$ which displaces $P$, it follows from Lemma 5 that $P \rho \in \pi_{0}$. Let $Q \neq P$ belong to $\pi_{0}$ and let $\tau$ be a collineation fixing $P$.

By Lemma 4, the line which contains $P$ and $Q \tau$ must belong to $\pi_{0}$. By Lemma 3, $\pi$ is a strict s.t. plane with respect to this line. This implies that $Q \tau \in \pi_{0}$ and we have established (1).

Part (2) follows from Lemmas 4 and 5.

Part (3) is a direct consequence of Lemma 3.

THEOREM 3. Under the hypothesis of Theorem 2, either (1) there is some point $P \in \pi_{0}$ which is fixed by all collineations of $\pi$ or (2) every elation of $\pi_{0}$ extends to an elation of $\pi$; every collineation in the little projective group of $\pi_{0}$ extends to a collineation on $\pi$.

Proof. The points of $\pi_{0}$ which are not in $l$ are all in a single transitive class under the group of elations with axis $l$. If there is a collineation which carries $l$ into $l_{1}$ and $l \cap l_{1}=P$, then all points of $\pi_{0}$ distinct from $P$ lie in a single transitive class under the full group of collineations. Thus if there is any collineation displacing $P$, all points of $\pi_{0}$ are in a single transitive class. But this implies [10] that all lines of $\pi_{0}$ are in a single transitive class.

The theorem then follows from the fact that $\pi$ is an s.t. plane with respect to every line of $\pi_{0}$.

We shall now be concerned with conditions under which a semi-translation plane may be self dual. More generally, we shall consider the case where $\pi$ is a semi-translation plane and also the dual of a semi-translation plane.

Definition. We shall say that $\pi$ is a dual semi-translation plane with respect to the point $Q$ if there exists a set $\mathfrak{N}$ of $q+1$ lines through $Q$ such that for each line $m \in \mathfrak{N}, \pi$ admits a group of elations of order $q$ with center $Q$ and axis $m$.

LEMMA 6. If $\pi$ is s.t. with respect to $l$ and is dual s.t. with respect to $Q$, where $Q \notin l$, then $\pi$ admits a collineation moving $l$. If $\pi$ is strict s.t. with respect to $l$, then $Q$ belongs to the invariant subplane $\pi_{0}$ of Theorem 2 . Every collineation of the little projective group of $\pi_{0}$ extends to a collineation of $\pi$. 
Proof. Since the elations with center $Q$ fix only the lines through $Q$, there is a collineation displacing $l$ and an invariant subplane $\pi_{0}$ in case $\pi$ is a strict s.t. plane with respect to $l$.

It is well known (and can easily be established by a counting argument) that if $\pi$ is any projective plane of order $q^{2}$ and $\pi_{0}$ is any subplane of order $q$, then every point of $\pi$ lies on at least one line of $\pi_{0}$.

Let $l_{1}$ be a line through $Q$ which belongs to $\pi_{0}$. If $Q \notin \pi_{0}, Q$ has an axis $l_{2}$ which does not belong to $\pi_{0}$. The group of elations with center $Q$ and axis $l_{2}$ permutes the $q^{2}+1$ points of $l_{1}$ in $q$ transitive classes of length $q, Q$ being the sole fixed point. Since the $q+1$ points on $l_{1} \cap \pi_{0}$ must be carried into themselves, one of these points must be fixed. That is, $Q$ must be a point on $l_{1}$ which belongs to $\pi_{0}$.

Note that $\pi$ admits collineations (elations with axis $l$ ) which displace $Q$. For each other point $T$ in $\pi_{0}$, there is an elation with center $Q$ whose axis does not go through $T$. Hence no point of $\pi_{0}$ is fixed by all collineations of $\pi$. Hence the last part of Lemma 6 follows from Theorem 3.

LEMMA 7. If $\pi$ is a strict s.t. plane with respect to $l$ and $\pi$ is a strict dual s.t. plane with respect to a point $Q$ on $l$, then $Q$ is a center for $l$. There exists $a$ subplane $\pi_{0}$ which contains the centers for $l$ and the axes for $Q$. If there is $a$ collineation of $\pi$ displacing either $l$ or $Q$, then there is an invariant subplane.

Proof. Since all collineations fixing $l$ must fix $\mathfrak{M}$ (Definition 1 ) and $\mathfrak{M}$ contains $q+1$ points, elations whose order divides $q$ must fix some point in $\mathfrak{M}$. Hence $Q$ must belong to $\mathfrak{M}$, i.e., $Q$ is a center for $l$.

If $Q$ is a center for $l$, the lines through $Q$ distinct from $l$ are split into transitive classes of length $q$ by the elation group $G(l)$. Thus the $q$ axes for $Q$ which are distinct from $l$ all lie in the same transitive class under $G(l)$.

If $R$ is any point not on $l$ but on an axis of $Q$, the subplane determined by $R$ as in Lemma 1 contains the centers for $l$ and the axes for $Q$.

The remainder of Lemma 7 follows from Theorem 2 and its dual.

THEOREM 4. If $\pi$ is a strict semi-translation plane with respect to $l$ and $\pi$ is self dual, then $\pi$ admits a duality in which l corresponds to one of its centers $Q$. Either $l$ and $Q$ are fixed by collineations of $\pi$ or there is a subplane $\pi_{0}$ such that $\pi_{0}$ is fixed by all collineations of $\pi$ and every collineation in the little projective group of $\pi_{0}$ extends to a collineation of $\pi$.

Proof. If $\pi$ admits a duality in which $l$ corresponds to a point $R$ not on $l$, Theorem 4 follows from Lemma 6 and Theorems 2 and 3, since the little projective group is transitive on points, i.e., there is a collineation carrying $R$ into any given point on $l$.

If every duality of $\pi$ makes $l$ correspond to a point on $l$, Theorem 4 follows from Lemma 7, Theorem 3 and its dual. Note that if some collineation moves $l$, there is a "dual" collineation moving $Q$. 
RemarK. Note that if $\pi$ is an s.t. plane with respect to $l$ but is not a strict s.t. plane with respect to $l$, the subplane of Lemma 1 is definitely not invariant-the elation group $G(l)$ is too large. Thus the "strictness " is essential to Theorems 2, 3, and 4, at least insofar as the invariant subplane is concerned. Most of the known s.t. planes are "strict." Note also that $\pi_{0}$ is Desarguesian if Theorem 2 applies.

III. Construction of planes. In this section we are not, at the outset, concerned with semi-translation planes. Theorem 5 (below) holds without restrictions on the order of the plane.

The following, pointed out to the author by D. R. Hughes, is very similar to Theorem 20.8.1 in [6]:

LEMMA 8. Let $\pi$ be a system consisting of $n^{2}>1$ points and certain specified sets of points, called lines, such that:

(1) Each line contains $n$ points.

(2) For each pair of distinct points $P$ and $Q$, there is exactly one line which contains $P$ and $Q$. Then $\pi$ is an affine plane.

Proof. Since the lines through a given point $P$ induce a partition of the $n^{2}-1$ remaining points into disjoint subsets each containing $n-1$ points, it follows that each point lies on exactly $n+1$ lines. If $P$ is not on the line $l, n$ of the lines through $P$ will intersect $l$ and the remaining line will be "parallel" to $l$.

In the rest of this paper, $\pi$ will denote an affine plane and $\pi^{*}$ will denote the projective plane obtained by adjoining a line $l_{\infty}$ to $\pi$. If $\pi^{*}$ is a semi-translation plane with respect to $l_{\infty}$, we shall say that $\pi$ is a semi-translation plane. Note that this is a reversal of our earlier notation.

THEOREM 5. Let $\pi$ be an affine plane of order $n$, let $\mathfrak{M}$ be a set of points on $l_{\infty}$ in $\pi^{*}$, and let $\mathfrak{S}$ be a class of sets of affine points such that:

(1) Each element of $\mathbb{S}$ contains exactly $n$ points.

(2) For each pair of affine points $P$ and $Q$ such that $P Q \cap l_{\infty} \in \mathfrak{M}$, there is exactly one element of $\mathbb{S}$ which contains $P$ and $Q$.

(3) If $P$ and $Q$ are distinct points which belong to the same element of $\mathfrak{S}$, then $P Q \cap l_{\infty} \in \mathfrak{M}$.

Then we can form a new affine plane $\bar{\pi}$, where the points of $\bar{\pi}$ are the points of $\pi$ and the lines of $\bar{\pi}$ are (1) the lines of $\pi$ whose extensions to $\pi^{*}$ contain no points of $\mathfrak{M}$; (2) the elements of $\mathfrak{S}$.

Proof. Each pair of points is contained in either one line of class (1) and no line of class (2) or in one line of class (1) but no line of class (2). Hence the theorem follows from Lemma 8.

In [8], the author considered the case where $\pi$ is Desarguesian; each set in $\widetilde{\Xi}$ was a transitive class of points under an appropriately selected subgroup of the translation group. In [9], $\pi$ was a dual translation plane, the sets in $\subseteq$ were subplanes of $\pi$. 
We now return to our restriction that all planes are of order $q^{2}$ and we consider only subplanes of order $q$. However, $q$ need not be assumed to be a prime power.

LeMma 9. Let $\pi^{*}$ be a projective plane of order $q^{2}$. Let $\mathfrak{M}$ be a set of $q+1$ points on $l_{\infty}$. If $P$ and $Q$ are two affine points such that the line $P Q$ contains $a$ point of $\mathfrak{M}$, then there is at most one projective subplane which contains $\mathfrak{M}$, $P$, and $Q$.

Proof. Let $\mathbb{E}$ be the set of points of intersection of the lines determined by $P$ and $\mathfrak{M}$ with the lines determined by $Q$ and $\mathfrak{M}$. Any subplane $\pi_{0}$ which contains $P, Q, \mathfrak{M}$ must contain all of the points of $\mathfrak{E}$. (Note that any proper subplane which contains $\mathfrak{M}$ must be of order $q$ since a plane of order $q^{2}$ can have no subplanes whose order is greater than $q$ and $\mathfrak{M}$ contains $q+1$ points.) Excepting the line $P Q$, we have determined $q+1$ points in $\pi_{0}$ on each of $q$ lines through $P$. That is, all of the points of $\pi_{0}$ are determined except those on $P Q$. But if $R$ is any point of $\mathbb{E}$ not on $P Q, R$ and $P$ determine the points on $P Q$. Hence the points of $\pi_{0}$ are completely determined.

THEOREM 6. Let $\pi$ be an affine plane of order $q^{2}$ and let $\mathfrak{M}$ be a set of $q+1$ points on $l_{\infty}$ on $\pi^{*}$. Suppose that for every pair of distinct points $P$ and $Q$ such that $P Q \cap l_{\infty} \in \mathfrak{M}$ there exists a projective subplane of $\pi^{*}$ which contains $P, Q$, and $\mathfrak{M}$. Then the affine parts of the proper subplane of $\pi^{*}$ which contain $\mathfrak{M}$ and the affine parts of the lines of $\pi^{*}$ which do not intersect $\mathfrak{M}$ form the lines of a new affine plane $\bar{\pi}$ containing the same points as $\pi$.

Proof. The proof is a direct consequence of Theorem 5 and Lemma 9.

Definition. Following Albert [1], a plane satisfying the conditions of Theorem 6 will be said to be derivable and $\pi$ will be said to be the derived plane.

COROLlary. In Theorem 6, the lines of $\pi$ which are not also lines of $\bar{\pi}$ are affine subplanes of $\bar{\pi}$. $\pi$ can be obtained from $\bar{\pi}$ in the same way that $\bar{\pi}$ was obtained from $\pi$.

Proof. Let $l$ be a line of $\pi$ but not a line of $\bar{\pi}$. Then $l$ contains $q^{2}$ points. For every pair of points $P, Q$ of $l$ there is exactly one line $l$ of $\bar{\pi}$ which contains $P$ and $Q$; moreover $l \cap l$ contains exactly $q$ points. Taking the set of points in $l \cap l$ as the set of points on a line of $\bar{\pi}_{0}$ (whose points are the points of $l$ ), it follows from Lemma 8 that $\bar{\pi}_{0}$ is an affine plane. Hence $\bar{\pi}_{0}$ is an affine subplane of $\bar{\pi}$. If $\bar{\pi}$ be extended to a projective plane $\bar{\pi}^{*}, q^{2}-q$ points at infinity will correspond to the parallel classes of the lines common to $\pi$ and $\bar{\pi}$. The remaining $q+1$ points at infinity in $\bar{\pi}^{*}$ will then be common to the extensions of the subplanes corresponding to the remaining lines of $\pi$, i.e., they will form the set $\overline{\mathfrak{M}}$. 
THEOREM 7. Let $\sigma$ be a permutation of the points of $\pi$ inducing a collineation of $\pi^{*}$ which carries $\mathfrak{M}$ into itself. Then $\sigma$ induces a collineation of $\bar{\pi}^{*}$ which carries $\overline{\mathfrak{M}}$ into itself.

Proof. Collineations of $\pi^{*}$ which fix $\mathfrak{M}$ carry the set of subplanes containing $\mathfrak{M}$ into itself. Thus $\sigma$ induces a collineation of $\bar{\pi}$. Any collineation of an affine plane can be extended to a collineation of the corresponding projective plane. The invariance of $\mathfrak{M}$ implies that the set of parallel classes of lines common to $\pi$ and $\bar{\pi}$ is carried into itself. This implies that the extension of $\sigma$ to $\bar{\pi}^{*}$ carries $\overline{\mathfrak{M}}$ into itself.

COROLlary. If $\sigma$ induces a translation of $\pi$, then $\sigma$ induces a translation of $\bar{\pi}$.

Proof. We need only consider the effect of $\sigma$ on the lines which are not common to $\pi$ and $\bar{\pi}$.

Let $P$ and $Q$ be two points such that $P Q \cap \mathfrak{M}=B$, where $P Q$ denotes a line of $\pi$. Let $A$ and $C$ belong to $\mathfrak{M}$. Let $Q C \cap P A=S, S B \cap Q A=T$. Then $P, Q, S$, $T$ all belong to a subplane $\pi_{0}$ of $\pi^{*}$ which contains $\mathfrak{M}$.

A translation of $\pi$ which carries $P$ into $Q$ must carry $S$ into $T$. Since $\pi_{0}$ is determined by $P, S$ and also by $Q, T$ (Lemma 9 ), the translation in question takes $\pi_{0}$ into itself. That is, the translation which carries $P$ into $Q$ fixes the subplane containing $P, Q$ and $\mathfrak{M}$; that is, it fixes the line of $\bar{\pi}$ which contains $P$ and $Q$.

Now let $\bar{l}_{0}$ and $l_{1}$ be lines of $\bar{\pi}$ such that $\bar{l}_{1}$ is the image of $\bar{l}_{0}$ under a translation $\tau$ of $\pi$. We must show that $\bar{l}_{1}$ is parallel to $l_{0}$. If $\pi_{0}^{*}$ and $\pi_{1}^{*}$ are the corresponding subplanes of $\pi^{*}$, this amounts to saying that the affine planes $\pi_{0}$ and $\pi_{1}$ have no points in common.

Suppose that $\pi_{0} \cap \pi_{1}=Q$ and $\pi_{0} \tau=\pi_{1}$. Then $\exists P \in \pi_{0} \ni P \tau=Q$. Since $P$ and $Q$ both belong to $\pi_{0}$, this can only happen (by the argument given above) if $\pi_{1}=\pi_{0}$. Hence either $\pi_{0}$ is fixed by $\tau$ or is "parallel" to its image. This completes the proof.

COROLlary. Let $l$ be a line of $\pi$ which is not a line of $\bar{\pi}$. If $\pi$ admits a group of translations transitive on the points of $l$, then $\bar{\pi}^{*}$ is a semi-translation plane with respect to $\bar{l}_{\infty}$. Conversely, if $\bar{\pi}$ is a semi-translation plane, there exists such a line $l$ in $\pi$.

IV. Coordinate systems. In this section, we shall be using a modification of Hall's ternary ring $[5 ; 6]$. That is, each point of the affine plane will be represented by ordered pairs $(x, y)$ of elements of an algebraic system $\mathfrak{T}$. Two binary relations, "addition" and "multiplication," are used with the same meanings that these terms have in the ternary. Lines will be represented by equations satisfied by the coordinates. $\mathfrak{I}$ will be said to be linear if every line has an equation of the form $y=x m+b$ or $x=c$. In the projective plane $\pi^{*}$ we shall denote the point at infinity on the line $y=x m$ by $(m)$, the point at infinity on $x=0$ by $(\infty)$. A line 
whose extension to $\pi^{*}$ contains $(m)$ will be said to have slope $m$. If $\mathfrak{F}$ is some subset of $\mathfrak{I}$ such that every line whose slope $\alpha$ belongs to $\mathfrak{F}$ has an equation of the form $y=x \alpha+\beta$, we shall say that $\mathfrak{T}$ is linear with respect to $\mathfrak{F}$.

Let $\mathfrak{F}$ be a subset of the elements of $\mathfrak{T}$. Suppose that, using the same operations as in $\mathfrak{I}$ and the same form for the equations of lines, $\mathfrak{F}$ is the coordinate system of a plane. Then we shall say that $\mathfrak{F}$ is a subsystem of $\mathfrak{T}$. Note that $\mathfrak{F}$ necessarily includes the elements 0 and 1 and is closed under the operations in $\mathfrak{T}$.

We shall be especially concerned with cases where $\mathfrak{I}$ is of order $q^{2}$ and contains a system $\mathfrak{F}$ of order $q$. In such cases, small Greek letters are to be understood as designating elements of $\mathfrak{F}$. The letters $\rho, \sigma$, and $\tau$ are exceptions and will be reserved to denote operators.

THEOREM 8. Let $\pi^{*}$ be a semi-translation plane. Let $\pi_{0}$ be an affine subplane of $\pi$ as in Lemma 1 . Then $\pi$ can be coordinatised by a system $\mathfrak{T}$ containing a subsystem $\mathfrak{F}$ which is a Veblen-Wedderburn system of order q. Furthermore:

(1) Points of $\pi_{0}$ have coordinates in $\mathfrak{F}$.

(2) Lines of $\pi_{0}$ have equations of the type $y=x \alpha+\beta$ or $x=\gamma$.

(3) Lines of $\pi$ whose slopes $m$ are not in $\mathfrak{F}$ have equations of the form: $y=(x-\alpha) m+\beta$.

(4) $(x-\alpha) \gamma=x \gamma-\alpha \gamma$ for all $x \in \mathfrak{T}$, all $\alpha, \gamma \in \mathfrak{F}$.

(5) If $c \notin \mathfrak{F}$ and $d$ is any element of $\mathfrak{I}$, there exist unique $\alpha$ and $\beta$ such that $d=c \alpha+\beta$.

Proof. Since $\pi_{0}$ is a translation plane, the coordinate system can be chosen so that $\pi_{0}$ is coordinatised by a Veblen-Wedderburn system $\mathfrak{F}$. It is readily verified that the translation which carries $(0,0)$ into $(\alpha, 0)$ is given by the mapping $(x, y) \rightarrow(x+\alpha, y)$. Similarly, the translation carrying $(0,0)$ onto $(0, \beta)$ is given by $(x, y) \rightarrow(x, y+\beta)$ and the translations of $G($ Lemma 1$)$ are $(x, y) \rightarrow(x+\alpha, y+\beta)$.

This implies that $(x+\alpha)+\beta=x+(\alpha+\beta)$ for all $x$ in $\mathfrak{T}$.

The fact that $\pi_{0}$ is of order $q$ and $\pi$ is of order $q^{2}$ implies that every line of $\pi^{*}$ contains at least one point of $\pi_{0}^{*}$. Thus if a line $l$ contains none of the points at $\infty$ of $\pi_{0}^{*}$, the slope $m$ of $l$ does not belong to $\mathfrak{F}$ and $l$ contains some point $(\alpha, \beta)$.

It follows that $l$ is the image of the line $y=x m$ under the translation $(x, y) \rightarrow(x+\alpha, y+\beta)$. This implies that coordinates of points on $l$ satisfy $y=(x-\alpha) m+\beta$.

The above equation still holds if $m \in \mathfrak{F}$ and the line $l$ goes through a point $(\alpha, \beta)$. In particular, a line of slope $\gamma, \gamma \in \mathfrak{F}$ through $(\alpha, 0)$ has the equation $y=(x-\alpha) \gamma$. Since this line also goes through $(0, \alpha \gamma)$, it also has the equation $y=x \gamma+\alpha \gamma$. Hence $(x-\alpha) \gamma=x \gamma-\alpha \gamma$ and, since the lines in question belong to $\pi_{0}$, lines of $\pi_{0}$ are coordinatised by equations of the type $y=x y+\beta$.

Every point of $\pi$ which is not in $\pi_{0}$ lies on exactly one line of $\pi_{0}$. Hence if $c \notin \mathfrak{F}$, the point $(c, d)$ lies on some line $y=x \alpha+\beta$, i.e., $d=c \alpha+\beta$. Conversely, if $d=c \alpha+\beta,(c, d)$ is on $y=x \alpha+\beta$. Hence $c$ and $d$ uniquely determine $\alpha$ and $\beta$. 
THEOREM 9. Let $\pi$ be an affine plane of order $q^{2}$ coordinatised by a system $\mathfrak{I}$ such that:

(1) $\mathfrak{I}$ contains a subsystem $\mathfrak{F}$ of order $q$.

(2) $\mathfrak{I}$ is linear with respect to $\mathfrak{F}$.

(3) Addition in $\mathfrak{I}$ is associative and commutative.

(4) For each $a \neq 0$ in $\mathfrak{I}$ any two of $\alpha, \beta, \gamma$ (in $\mathfrak{F}$ ) uniquely determines the third so that $a \alpha-a \beta=a \gamma$.

(5) Similarly, any two of $\alpha, \beta, \gamma$ determine the third so that $(a \alpha) \beta=a \gamma$.

(6) For given $a, b \in \mathfrak{I}$ such that $a+b \neq 0, \alpha$ and $\beta$ uniquely determine each other by the equation

$$
(a+b) \alpha=a \beta+b \beta .
$$

Then, (1) for each set of fixed elements $a \neq 0, c, d$ of $\mathfrak{T}$, the set $\mathfrak{R}$ of points of the form $(a \xi+c, a \eta+d)($ where $\xi$ and $\eta$ range over $\mathfrak{F})$ is the set of points in an affine subplane. (2) $\pi$ is derivable.

Proof. Note that the number of points in $\Re$ is equal to $q^{2}$, since $\xi$ and $\eta$ may independently take on $q$ different values each.

Let $P=\left(a \xi_{1}+c, a \eta_{1}+d\right), Q=\left(a \xi_{2}+c, a \eta_{2}+d\right)$. If $\xi_{1}=\xi_{2}, P$ and $Q$ are on

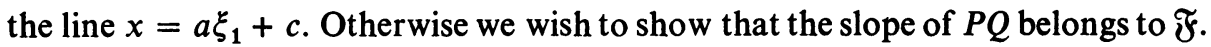

It will suffice to show that there exist $\mu, b$ such that $P$ and $Q$ are both on $y=x \mu+b$ (hypothesis (1)). This will be the case if there exists $\mu$ such that $a \eta_{1}-a \eta_{2}=\left(a \xi_{1}+c\right) \mu-\left(a \xi_{2}+c\right) \mu$.

Let $\xi$ be determined so that $a \xi_{1}-a \xi_{2}=a \xi$. Let $\eta$ be determined so that $a \eta_{1}-a \eta_{2}=a \eta$. Let $\gamma$ be determined so that $(a \xi) \gamma=a \eta$. Let $\mu$ be determined so that

Then

$$
\left(a \xi_{1}+c\right) \mu-\left(a \xi_{2}+c\right) \mu=\left[\left(a \xi_{1}+c\right)-\left(a \xi_{2}+c\right)\right] \gamma
$$

$$
\left(a \xi_{1}+c\right) \mu-\left(a \xi_{2}+c\right) \mu=\left(a \xi_{1}-a \xi_{2}\right) \gamma=(a \xi) \gamma=a \eta=a \eta_{1}-a \eta_{2} .
$$

Hence the slope of $P Q$ belongs to $\mathfrak{F}$.

Now the equation of $P Q$ may be written

$$
y=x \mu+\left(a \eta_{1}+d\right)-\left(a \xi_{1}+c\right) \mu .
$$

If we put $x=a \xi+c$ ( $\xi$ not necessarily the same as determined above), we get

$$
\begin{aligned}
y & =(a \xi+c) \mu+\left(a \eta_{1}+d\right)-\left(a \xi_{1}+c\right) \mu \\
& =\left[(a \xi+c)-\left(a \xi_{1}+c\right)\right] \lambda+a \eta_{1}+d \text { for some } \lambda \\
& =\left(a \xi-a \xi_{1}\right) \lambda+a \eta_{1}+d \\
& =(a v) \lambda+a \eta_{1}+d \text { for some } v \\
& =a \eta+d \text { for some } \eta
\end{aligned}
$$


That is, for each $\xi$, there exists a unique $\eta$ such that $(a \xi+c, a \eta+d)$ is on the line $P Q$. Thus $P Q \cap \Re$ contains exactly $q$ points.

Consider the geometrical system whose points are the points of $\mathfrak{R}$ and whose lines are the intersections of $\Re$ with the lines of $\pi$ containing at least two points of $\mathfrak{R}$. By Lemma 8 , this system is an affine plane. This establishes conclusion (1).

We now wish to apply Theorem 6 . The set $\mathfrak{M}$ consists of $(\infty)$ and $(\alpha)$, where $\alpha$ varies over $\mathfrak{F}$. We must establish that if $P$ and $Q$ are any two points such that the line $P Q$ intersects $l_{\infty}$ in $\mathfrak{M}$, then there exist $a, c, d, \xi_{1}, \eta_{1}, \xi_{2}, \eta_{2}$ such that $P=\left(a \xi_{1}+c, a \eta_{1}+d\right), \quad Q=\left(a \xi_{2}+c, a \eta_{2}+d\right)$.

Suppose that $P$ and $Q$ are on the line $y=x \mu+b$. We may write

$$
P=\left(x_{1}, x_{1} \mu+b\right), \quad Q=\left(x_{2}, x_{2} \mu+b\right), \quad x_{1} \neq x_{2} .
$$

Let $\eta_{2}$ be determined so that

$$
x_{2} \mu-x_{1} \mu=\left(x_{2}-x_{1}\right) \eta_{2} .
$$

Let $a=x_{2}-x_{1}, c=x_{1}, \xi_{1}=0, \xi_{2}=1, d=x_{1} \mu+b$. Then the coordinates of $P$ and $Q$ are in the desired form. We now apply Theorem 6 to complete the proof of our theorem.

Note that, as in part (5) of Theorem 8, if $t$ is some fixed element of $\mathfrak{I}$ which is not in $\mathfrak{F}$ and $a$ is any element of $\mathfrak{I}$, then the point $(t, a)$ is on exactly one line $y=x \alpha+\beta$ of the subplane coordinatised by $\mathfrak{F}$. Hence there exist $\alpha$ and $\beta$ such that $a=t \alpha+\beta$.

In the known planes which are derivable (or, rather, the planes known to be derivable) $\mathfrak{F}$ is a field and conditions (4), (5), and (6) of Theorem 9 occur in the more restrictive form equivalent to saying that the additive group of $\mathfrak{I}$ is a vector space of dimension two over $\mathfrak{F}$. This situation will be denoted more briefly by saying that $\mathfrak{I}$ is a vector space over $\mathfrak{F}$.

The author would conjecture that systems satisfying the weaker conditions do exist. However, he has not been able to find any such systems.

The following two theorems are modifications of an argument due to Albert [1].

THEOREM 10. Let $\pi$ be an affine plane coordinatised by a system $\mathfrak{I}$ as in Theorem 9 , where $\mathfrak{T}$ is a vector space over $\mathfrak{F}$. Let $t$ be a fixed element of $\mathfrak{I}$ which is not in $\mathfrak{F}$. Then $\bar{\pi}$ can be coordinatised by a coordinate system $\overline{\mathfrak{T}}$ such that a point with coordinates $(x, y)=\left(t \xi_{1}+\xi_{2}, t \eta_{1}+\eta_{2}\right)$ in $\mathfrak{I}$ has coordinates $(\bar{x}, \bar{y})=\left(t \xi_{1}+\eta_{1}, t \xi_{2}+\eta_{2}\right)$ in $\overline{\mathfrak{I}}$.

Proof. Recall that, for fixed $a, c, d$, the set of points $(x, y)=(a \xi+c, a \eta+d)$ is a line of $\bar{\pi}$. Choose the coordinate system for $\bar{\pi}$ so that the following sets of points $(x, y)$ are respectively the lines $\bar{x}=0, \bar{y}=0$ and $\bar{y}=\bar{x}$ :

$$
(\xi, \eta),(t \xi, t \eta) \text { and }((t+1) \xi,(t+1) \eta) .
$$


Let $(x, y)=(0, t+1)$ be the point $(\bar{x}, \bar{y})=(1,1)$.

Then the points on $\bar{x}=$ constant must be the set $(x, y)=(\xi+c, \eta+d)$ for some fixed $(c, d)$.

The set of points on $\bar{y}=$ constant must be the set $(x, y)=(t \xi+c, t \eta+d)$ for some fixed $(c, d)$.

Now the point $(x, y)=\left(t \xi_{1}+\xi_{2}, t \eta_{1}+\eta_{2}\right)$, where $\xi_{1}, \xi_{2}, \eta_{1}, \eta_{2}$ are fixed, is the intersection of the sets for which

and

$$
(x, y)=\left(\xi+t \xi_{1}, \eta+t \eta_{1}\right) \quad(\xi, \eta \text { vary })(\text { i.e., } \bar{x} \text { is constant })
$$

$$
(x, y)=\left(t \xi+\xi_{2}, t \eta+\eta_{2}\right) \quad(\xi, \eta \text { vary) (i.e., } \bar{y} \text { is constant). }
$$

The respective intersections of these sets with $\bar{y}=\bar{x}$ are

$$
\left(\xi_{1}+t \xi_{1}, \eta_{1}+t \eta_{1}\right) \text { and }\left(t \xi_{2}+\xi_{2}, t \eta_{2}+\eta_{2}\right)
$$

Hence if we assign to the point $(x, y)=(t \xi+\xi, t \eta+\eta)$ on $\bar{y}=\bar{x}$ coordinates

then the set for which

$$
(\bar{x}, \bar{y})=\left(t \xi+\eta, t \xi_{i}+\eta\right)
$$

$$
(x, y)=\left(\xi+t \xi_{1}, \eta+t \eta_{1}\right)
$$

becomes $\bar{x}=t \xi_{1}+\eta_{1}$. Similarly,

$$
(x, y)=\left(t \xi+\xi_{2}, t \eta+\eta_{2}\right)
$$

becomes $\bar{y}=t \xi_{2}+\eta_{2}$. That is, if

$$
(x, y)=\left(t \xi_{1}+\xi_{2}, t \eta_{1}+\eta_{2}\right),
$$

then $(\bar{x}, \bar{y})=\left(t \xi_{1}+\eta_{1}, t \xi_{2}+\eta_{2}\right)$.

THEOREM 11. Under the hypothesis of Theorem 10:

(1) Addition in $\overline{\mathfrak{T}}$ is isomorphic to addition in $\mathfrak{T}$.

(2) $\mathfrak{F}$ is a subsystem of $\overline{\mathfrak{I}}$.

(3) $\widetilde{T}$ is linear with respect to $\mathfrak{F}$.

(4) Multiplication on the right by elements of $\mathfrak{F}$ is the same in $\mathfrak{T}$ and $\overline{\mathfrak{T}}$.

(5) If $\circ$ denotes multiplication in $\overline{\mathfrak{T}}$, then $\left(t \xi_{1}+\eta_{1}\right) \circ\left(t \lambda_{1}+\lambda_{2}\right)=t \xi_{2}+\eta_{2}$ is equivalent to $\left(t \xi_{1}+\xi_{2}\right)\left(t \mu_{1}+\mu_{2}\right)=t \eta_{1}+\eta_{2}$ where $\lambda_{1}\left(t \mu_{1}+\mu_{2}\right)=t+\lambda_{2}$ provided $\lambda_{1} \neq 0$.

Proof. The set of points $(\bar{x}, \bar{y})=\left(\eta_{1}, \eta_{2}\right)$ is the set of points on $x=0$ and hence is a subplane of $\bar{\pi}$. Hence $\mathfrak{F}$ is a subsystem of $\overline{\mathfrak{T}}$.

In general if $l$ is a line of $\bar{\pi}$ but not a line of $\pi, l$ contains a set of points of the form

$$
(x, y)=\left((t \alpha+\beta) \xi+\left(t \gamma_{1}+\delta_{1}\right),(t \alpha+\beta) \eta+\left(t \gamma_{2}+\delta_{2}\right)\right)
$$

where $\xi$ and $\eta$ vary. For each $(x, y)$, the corresponding $(\bar{x}, \bar{y})$ are given by 


$$
\begin{aligned}
& \bar{x}=t\left(\alpha \xi+\gamma_{1}\right)+\left(\alpha \eta+\gamma_{2}\right), \\
& \bar{y}=t\left(\beta \xi+\delta_{1}\right)+\left(\beta \eta+\delta_{2}\right) .
\end{aligned}
$$

Hence $\bar{y}-\bar{x} \alpha^{-1} \beta=t\left(\delta_{1}-\gamma_{1} \alpha^{-1} \beta\right)+\left(\delta_{2}-\gamma_{2} \alpha^{-1} \beta\right)$ unless $\alpha=0$. For $\alpha=0, \bar{x}$ is independent of $\xi$, $\eta$, i.e., we have the line $\bar{x}=t \gamma_{1}+\gamma_{2}$. Thus the "new" lines are linear in $\overline{\mathfrak{I}}$ with slopes in $\mathfrak{F}$. This includes all lines of $\bar{\pi}$ with slopes in $\mathfrak{F}$, so we have established (3).

With the proper choice of $\delta_{1}, \gamma_{1}, \delta_{2}, \gamma_{2}$ we get that $\bar{y}=\bar{x} \alpha^{-1} \beta$ is the equation of the line of $\bar{\pi}$ through $(0,0)$ and $(\bar{x}, \bar{y})=\left(1, \alpha^{-1} \beta\right)$. This establishes (4).

Furthermore, with $\alpha=\beta$ we obtain $\bar{y}=\bar{x}+t\left(\delta_{1}-\gamma_{1}\right)+\left(\delta_{2}-\gamma_{2}\right)$ as the equation of the line in $\bar{\pi}$ with slope 1 through $(\bar{x}, \bar{y})=\left(0, t\left(\delta_{1}-\gamma_{1}\right)+\left(\delta_{2}-\gamma_{2}\right)\right)$. This is essentially the definition of addition in $\mathfrak{I}$; we have established (1).

Now let us determine multiplication in $\overline{\mathfrak{T}}$. The line through $(0,0)$ and $(\bar{x}, \bar{y})=\left(1, t \lambda_{1}+\lambda_{2}\right)$ has the equation $\bar{y}=\bar{x} \circ\left(t \lambda_{1}+\lambda_{2}\right)$. Now $(\bar{x}, \bar{y})=\left(1, t \lambda_{1}+\lambda_{2}\right)$ is equivalent to $(x, y)=\left(\lambda_{1}, t+\lambda_{2}\right)$. For $\lambda_{1} \neq 0$, this is also a line of $\pi$ through the origin. Its equation is of the form $y=x\left(t \mu_{1}+\mu_{2}\right)$, where $\lambda_{1}\left(t \mu_{1}+\mu_{2}\right)=t+\lambda_{2}$.

Conclusion (5) then follows from the general relation between $(x, y)$ and $(\bar{x}, \bar{y})$.

Note that in Theorem $11, x=0$ is the subplane coordinatised by $\mathfrak{F}$ in $\bar{\pi}$ and $\bar{x}=0$ is the subplane coordinatised by $\mathfrak{F}$ in $\pi$. In the more general situation, we can choose the coordinate systems so that $x=0$ is coordinatised by a subsystem $\overline{\mathfrak{F}}$ of $\overline{\mathfrak{T}}$ and $\bar{x}=0$ is coordinatised by a subsystem $\mathfrak{F}$ of $\mathfrak{I}$. This can be done without reference even to Theorem 9 , which has not been established as a necessary condition for derivability.

THEOREM 12. Let $\pi$ be a derivable semi-translation plane, where the set $\mathfrak{M}$ of Definition 1 coincides with the set $\mathfrak{M}$ of Theorem 6. Let $\bar{\pi}$ be coordinatised so that $\bar{x}=0$ is the affine part of one of the subplanes of Lemma 1. Then $\overline{\mathfrak{T}}$ is linear with associative and commutative addition.

Proof. By Theorem 20.4.3 in [6], the translation group of $\pi$ is abelian. Theorem 12 then follows by applying the corollaries to Theorem 7 and Theorem 20.4.5 in $[6]$.

LEMMA 10. Let $\pi$ be derivable and let $l$ be a line of $\pi$ which is a subplane of $\bar{\pi}$. Let $\bar{\pi}$ be coordinatised so that $l$ is coordinatised by a substem $\overline{\mathfrak{F}}$ of $\overline{\mathfrak{T}}$. Let $\sigma$ be a collineation of $\pi^{*}$ which fixes $l$ pointwise and carries $\mathfrak{M}$ into itself. Then $\sigma$ induces an automorphism of $\overline{\mathfrak{T}}$ which fixes each element of $\overline{\mathfrak{F}}$.

Proof. By Theorem $7 \sigma$ induces a collineation of $\pi$. This collineation fixes pointwise the subplane coordinatised by $\overline{\mathfrak{F}}$. Such a collineation induces an automorphism of $\overline{\mathfrak{I}}$ which fixes each element of $\overline{\mathfrak{F}}$.

LEMMA 11. Let $\pi$ be an affine plane coordinatised by a system $\mathfrak{I}$ containing a subsystem $\mathfrak{F}$. Suppose that $\pi$ admits all elations with axis $x=0$, center $(\infty)$ 
which carry $(0)$ into $(\alpha)$ for all $\alpha$ in $\mathfrak{F}$. Then $\mathfrak{I}$ satisfies the partial distributive law $a(b+\alpha)=a b+a \alpha$ for all $a, b$ in $\mathfrak{I}$ and all $\alpha$ in $\mathfrak{F}$.

Proof. The proof is a trivial modification of the argument used in the proof of part 2.3 of Theorem 20.5.2 in [6].

THEOREM 13. In Theorem 12, suppose that $\pi$ is a strict semi-translation plane which admits a collineation moving $l_{\infty}$ or is self dual. Then $\overline{\mathfrak{T}}$ can be chosen so that $\overline{\mathfrak{I}}$ also admits a group of automorphisms of order $q$ leaving the elements of $\overline{\mathfrak{F}}$ fixed.

Proof. The proof follows from Lemma 10, part 2 of Theorem 2, and Theorem 4.

THEOREM 14. Let $\pi$ be a derivable plane which is a dual translation plane with respect to a point in the set $\mathfrak{M}$ of Theorem 6 . Then $\bar{\pi}$ is a semi-translation plane which can be coordinatised by a system $\overline{\mathfrak{I}}$ satisfying the conditions of Theorem 8 and admitting a group of automorphisms of order $q$ which leaves elements of $\overline{\mathfrak{F}}$ fixed. If $\bar{\pi}$ is a strict semi-translation plane which is self dual, this can be done in such a way that $\overline{\mathfrak{T}}$ satisfies the partial distributive law

$$
a(b+\alpha)=a b+a \alpha .
$$

Proof. The proof follows from Lemmas 10 and 11, Theorems 2 and 4.

With the possible exception of some of the translation planes, the known semitranslation planes are all derivable and satisfy the hypotheses of Theorem 10 . Those constructed by the author [9] were derived from dual translation planes. They are strict semi-translation planes unless the dual translation plane has a translation group whose order is greater than $q^{2}$. A question of fundamental importance, therefore, is the nature of coordinate systems satisfying at least the partial left distributive law and admitting automorphisms as in Theorems 13 and 14.

THEOREM 15. Let $\pi$ be a semi-translation plane coordinatised as in Theorem 8 , where $\mathfrak{I}$ is a vector space over $\mathfrak{F}$. Suppose that $\mathfrak{T}$ admits a one-to-one mapping $\sigma$ onto itself and that

(1) $\mathfrak{I}$ is linear with respect to $\mathfrak{F}$.

(2) $a(b+\alpha)=a b+a \alpha$.

(3) $\alpha a=a \alpha$.

(4) $\alpha \sigma=\alpha$.

(5) $(a \alpha+\beta) \sigma=(a \sigma) \alpha+\beta$.

(6) $a(b \sigma) \in \mathfrak{F} \Rightarrow b(a \sigma)=a(b \sigma)$.

(7) $a \sigma^{2}=a$

for all $a, b$, in $\mathfrak{I}$ and all $\alpha, \beta$ in $\mathfrak{F}$.

Then $\pi$ is self dual.

Proof. Consider the following mapping of order two between points and lines: 


$$
\begin{aligned}
(\infty) \leftrightarrow l_{\infty}, & \\
(c \sigma) \leftrightarrow x & \leftrightarrow c, \\
(c, c \alpha-\beta) & \leftrightarrow y=(x-\alpha)(c \sigma)+\beta, \quad c \notin \mathfrak{F}, \\
(\alpha, d) & \leftrightarrow y=x \alpha-d \sigma .
\end{aligned}
$$

Such a mapping between points and lines is a polarity if, for every two points $P$ and $Q$ such that $P$ is on the polar of $Q$, then $Q$ is on the polar of $P$. (Note that, by Theorem $8, c \alpha-\beta$ takes on all values in $\mathfrak{I}$ as $\alpha, \beta$ vary and $c$ is fixed.)

If $P$ is a point at infinity and $Q$ is any other point, the verification of this property is immediate.

The condition that $(c, c \alpha-\beta)$ be on the polar of $(\gamma, d)$ is that $c \alpha-\beta=c \gamma-d \sigma$, i.e., $d \sigma=c(\gamma-\alpha)+\beta$. The condition that $(\gamma, d)$ be on the polar of $(c, c \alpha-\beta)$ is that $d=(\gamma-\alpha)(c \sigma)+\beta$. Under the hypotheses, each of these implies the other.

Let $t$ be a fixed element of $\mathfrak{I}$. By (5) of Theorem 8, each element of $\mathfrak{I}$ may be written in the form $t \alpha+\beta$.

The condition that $\left(t \xi_{1}+\xi_{2}+\alpha, t \gamma_{1}+\gamma_{2}+\beta\right)$ be on the polar of $\left(t \eta_{1}+\eta_{2},\left(t \gamma_{1}+\gamma_{2}\right) a-\beta\right)$ is that $\left(t \xi_{1}+\xi_{2}\right)\left[\left(t \eta_{1}+\eta_{2}\right) \sigma\right]=t \gamma_{1}+\gamma_{2}$. We are reduced to the case already covered unless $\xi_{1}$ and $\eta_{1}$ are both different from zero.

Now $t \gamma_{1}+\gamma_{2}+\beta=\left(t \xi_{1}+\xi_{2}+\alpha\right) \xi_{1}^{-1} \gamma_{1}-\left(\xi_{2} \xi_{1}^{-1} \gamma_{1}+\alpha \xi_{1}^{-1} \gamma_{1}-\gamma_{2}-\beta\right)$.

Hence the polar of $\left(t \xi_{1}+\xi_{2}+\alpha, t \gamma_{1}+\gamma_{2}+\beta\right)$ is

$$
y=\left(x-\xi_{1}^{-1} \gamma_{1}\right)\left[\left(t \xi_{1}+\xi_{2}+\alpha\right) \sigma\right]+\xi_{2} \xi_{1}^{-1} \gamma_{1}+\alpha \xi_{1}^{-1} \gamma_{1}-\gamma_{2}-\beta .
$$

Hence we must show the equivalence of the conditions

$$
\begin{gathered}
\left(t \xi+\xi_{2}\right)\left[\left(t \eta_{1}+\eta_{2}\right) \sigma\right]=t \gamma_{1}+\gamma_{2}, \\
\left(t \eta_{1}+\eta_{2}-\xi_{1}^{-1} \gamma_{1}\right)\left[\left(t \xi_{1}+\xi_{2}+\alpha\right) \sigma\right]+\xi_{2} \xi_{1}^{-1} \gamma_{1}+\alpha \xi_{1}^{-1} \gamma_{1}-\gamma_{2}-\beta \\
=t \eta_{1} \alpha+\eta_{2} \alpha-\beta .
\end{gathered}
$$

The second equation reduces to

$$
\left(t \eta_{1}+\eta_{2}-\xi_{1}^{-1} \gamma_{1}\right)\left[\left(t \xi_{1}+\xi_{2}\right) \sigma\right]=\gamma_{2}-\xi_{2} \xi_{1}^{-1} \gamma_{1} .
$$

By condition (6), this is equivalent to

$$
\left(t \xi_{1}+\xi_{2}\right)\left[\left(t \eta_{1}+\eta_{2}-\xi_{1}^{-1} \gamma_{1}\right) \sigma\right]=\gamma_{2}-\xi_{2} \xi_{1}^{-1} \gamma_{1}
$$

or

$$
\left(t \xi_{1}+\xi_{2}\right)\left[\left(t \eta_{1}+\eta_{2}\right) \sigma\right]-\left(t \xi_{1}+\xi_{2}\right) \xi_{1}^{-1} \gamma_{1}=\gamma_{2}-\xi_{2} \xi_{1}^{-1} \gamma_{1}
$$

i.e.,

$$
\left(t \xi_{1}+\xi_{2}\right)\left[\left(t \eta_{1}+\eta_{2}\right) \sigma\right]=t \gamma_{1}+\gamma_{2}
$$

We have taken care of all cases except those in which the $x$ coordinates of both $P$ and $Q$ belong to $\mathfrak{F}$. The condition that $(\gamma, c)$ be on the polar of $(\alpha, d)$ is that 
$c=\gamma \alpha-d \sigma$; that $(\alpha, d)$ be on the polar of $(\gamma, c)$ is that $d=\alpha \gamma-c \sigma$. These two are equivalent.

V. Special cases. If $\pi^{*}$ is a Hughes plane [7], then $\pi^{*}$ is of order $q^{2}$ and has a subplane $\pi_{0}^{*}$ of order $q$. Each collineation in the projective group of $\pi_{0}^{*}$ extends to a collineation of $\pi^{*} ; \pi_{0}^{*}$ is invariant under all collineations of $\pi^{*}$. Thus $\pi^{*}$ is a strict semi-translation plane with respect to every line of $\pi_{0}^{*}$.

The homogeneous coordinate system used by Hughes becomes, in the nonhomogeneous form, a coordinate system satisfying the conditions of Theorem 8 . Multiplication and addition in $\mathfrak{I}$ are the same as in a left nearfield of order $q^{2} . \mathfrak{I}$ is a vector space over $\mathfrak{F}$; the elements of $\mathfrak{F}$ commute in the multiplicative group of $\mathfrak{I}$. It readily follows that the conditions of Theorem 15 are satisfied with $\sigma$ being the identity. Hence the Hughes planes are self dual.

Hughes has pointed out to the author that the Hughes planes are derivable. Indeed, Theorems 10 and 11 apply. Let $\bar{\pi}$ be the plane derived from a Hughes plane. Since the order of the translation group of the affine Hughes plane is exactly $q^{2}$, it follows from Theorem 7 and its corollaries that the translation group of $\bar{\pi}$ has exactly one center; the affine points on each line through this point at infinity constitute a single transitive class. (If we use Theorems 10 and 11 to coordinatise $\bar{\pi}$, these will be the lines $\bar{x}=$ constant.) Hence $\bar{\pi}$ is not a semi-translation plane. The line $\bar{x}=0$ is fixed by collineations fixing $\overline{\mathfrak{M}}$. If $\bar{\pi}$ is a dual translation plane, it must admit allelations with axis $\bar{x}=0$, center $(\bar{\infty})$. This implies that the near field coordinatising $\pi$ has a group of automorphisms of order $q$. With certain possible exceprions, nearfields of order $q^{2}$ do not admit such automorphisms [14].

Hence the planes derived from the Hughes planes form another new class of planes.

Another interesting special case is the following. Suppose that we start out with a Desarguesian plane coordinatised by a field and apply Theorems 10 and 11 . We obtain a right Veblen-Wedderburn system. (Hughes and Albert [1] have both pointed out that this is a Hall V. W. system.)

Interchanging left and right multiplication, we get a left $\mathrm{V}$. W. system to which Theorems 10 and 11 can again be applied to get the coordinate system of a semitranslation plane.

Specifically, if the original field was of order $q^{2}$, if $\mathfrak{F}$ was a subfield order $q$ and $t$ was an element not in the subfield such that $t^{2}=t \alpha_{0}+\beta_{0}$, then multiplication in the final system (coordinatising the semi-translation plane) is given as follows:

$$
\begin{aligned}
\left(t \xi_{1}+\xi_{2}\right)\left(t \lambda_{1}+\lambda_{2}\right)= & t\left(\lambda_{2} \xi_{1}-\xi_{2} \lambda_{1}-\alpha_{0}\right) \\
& \quad+\left(\lambda_{2} \xi_{2}-\alpha_{0} \xi_{2} \xi_{1}^{-1}-\xi_{2}^{2} \xi_{1}^{-1} \lambda_{1}+\lambda_{1}^{-1} \xi_{1}^{-1} \beta_{0}\right), \quad \xi_{1}, \lambda_{1} \neq 0, \\
\gamma\left(t \lambda_{1}+\lambda_{2}\right)= & \left(t \lambda_{1}+\lambda_{2}\right) \gamma=t \lambda_{1} \gamma+\lambda_{2} \gamma .
\end{aligned}
$$


If we define $\sigma$ by $\left.\left(t \lambda_{1}+\lambda_{2}\right) \sigma\right]=-t \lambda_{1}+\lambda_{2}$, Theorem 15 applies and we have a self-dual semi-translation plane.

We have verified that for $\alpha_{0}=0, q \neq 3$, there are no collineations moving $l_{\infty}$ into $x=0$. The details are messy, and we omit the proof.

Finally, suppose that $\alpha_{0} \neq 0$ and $\mathfrak{F}$ is of characteristic two. We find that $\left(t \xi_{1}+\xi_{2}+\alpha_{0} \lambda_{1}^{-1}\right)\left(t \lambda_{1}+\lambda_{2}\right)=t \alpha_{0}+\alpha_{0} \lambda_{1}^{-1} \lambda_{2}+\left(t \xi_{1}+\xi_{2}\right)\left(t \lambda_{1}+\lambda_{2}\right)$. This implies that if $\left(t \xi_{1}+\xi_{2}+\alpha,\left(t \xi_{1}+\xi_{2}\right)\left(t \lambda_{1}+\lambda_{2}\right)+\beta\right)=(c, d)$ is on the line $y=(x-\alpha)\left(t \lambda_{1}+\lambda_{2}\right)+\beta$, then $\left(c, d+t \alpha_{0}\right)$ is on the line

$$
y=\left(x-\alpha-\alpha_{0} \lambda_{1}^{-1}\right)\left(t \lambda_{1}+\lambda_{2}\right)+\left(\beta+\alpha_{0} \lambda_{1}^{-1} \lambda_{2}\right) \text {. }
$$

(The cases where $\xi_{1}$ or $\lambda_{1}$ are zero require special treatment.)

Hence the mapping $(x, y) \rightarrow\left(x, y+t \alpha_{0}\right)$ is a translation in addition to the translations $(x, y) \rightarrow(x+\alpha, y+\beta)$.

Together with the dual translation planes from which they were derived, these planes form the only examples known to us of semi-translation planes which are neither translation planes nor strict semi-translation planes. André [3] has given examples of infinite planes with a similar property.

\section{REFERENCES}

1. A. A. Albert, The finite planes of Ostrom (unpublished).

2. J. André, Uber nicht-Desarguesche Ebenen mit transitiver translationsgruppe, Math Z. 60 (1954), 156-186.

3. — Affine Ebenen mit genugend vielen Translationen, Math. Nachr. 19(1958), 203-210.

4. A. M. Gleason, Finite Fano planes, Amer. J. Math. 78 (1956), 797-807.

5. M. Hall, Projective planes, Trans. Amer. Math. Soc. 54 (1943), 229-277.

6. - The theory of groups, Macmillan, New York, 1959.

7. D. R. Hughes, A class of non-Desarguesian projective planes, Canad. J. Math. 9 (1957), 378-388.

8. T. G. Ostrom. Translation planes and configurations in Desarguesian planes, Arch. Math. 11 (1960), 457-464.

9. —_ A class of non-Desarguesian affine planes, Trans. Amer. Math. Soc. 104 (1962), 483-487.

10. E. T. Parker, On collineations of symmetric designs, Proc. Amer. Math. Soc. 8 (1957), 350-351.

11. G. Pickert, Projective Ebenen, Springer, Berlin, 1955. 505-513.

12. L. Rosati, I gruppe collineazione dei piani di Hughes, Boll. Un. Mat. Ital. 13 (1958),

13. G. Zappa, Sui gruppi di collineazioni dei piani di Hughes, Boll. Un. Mat. Ital. 12 (1957), 507-516.

14. H. Zassenhaus, Über endliche Fastkörper, Abh. Math. Sem. Univ. Hamburg 11 (1935) 187-220.

WASHington STATE University, Pullman, Washington 